\title{
Genome sequence and global sequence variation map with 5.5 million SNPs in Chinese rhesus macaque
}

\author{
Xiaodong Fang ${ }^{1 \dagger}$, Yanfeng Zhang ${ }^{2,3 \dagger}$, Rui Zhang ${ }^{2,4 \dagger}$, Lixin Yang ${ }^{2,3}$, Ming Li ${ }^{2,3}$, Kaixiong Ye ${ }^{2}$, Xiaosen Guo ,
} Jun Wang ${ }^{1 *}$ and Bing Su 2 $^{*}$

\begin{abstract}
Background: Rhesus macaque (Macaca mulatta) is the most widely used nonhuman primate animal in biomedical research. A global map of genetic variations in rhesus macaque is valuable for both evolutionary and functional studies.

Results: Using next-generation sequencing technology, we sequenced a Chinese rhesus macaque genome with 11.56 -fold coverage. In total, $96 \%$ of the reference Indian macaque genome was covered by at least one read, and we identified 2.56 million homozygous and 2.94 million heterozygous SNPs. We also detected a total of 125,150 structural variations, of which 123,610 were deletions with a median length of $184 \mathrm{bp}$ (ranging from $25 \mathrm{bp}$ to 10 $\mathrm{kb}) ; 63 \%$ of these deletions were located in intergenic regions and 35\% in intronic regions. We further annotated 5,187 and 962 nonsynonymous SNPs to the macaque orthologs of human disease and drug-target genes, respectively. Finally, we set up a genome-wide genetic variation database with the use of Gbrowse.
\end{abstract}

Conclusions: Genome sequencing and construction of a global sequence variation map in Chinese rhesus macaque with the concomitant database provide applicable resources for evolutionary and biomedical research.

\section{Background}

Rhesus macaque (Macaca mulatta) and human shared a most recent common ancestor about 25 million years ago [1] and their genome sequences share $93.5 \%$ identity [2]. Due to the genetic and physiologic similarity between rhesus macaque and human, rhesus macaques are the most widely used nonhuman primate animals for biomedical research, for example, in vaccine development and as animal models for human diseases [3-7]. In research, rhesus macaque subspecies from India and China are the most commonly used, and the divergence between these was estimated to be about 162,000 years ago [8]. The observed genetic divergence, though shallow, is considered to underlie the observed phenotypic

\footnotetext{
* Correspondence: wangj@genomics.org.cn; sub@mail.kiz.ac.cn

† Contributed equally

'Beijing Genomics Institute-Shenzhen, Chinese Academy of Sciences, Shenzhen 518083, China

${ }^{2}$ State Key Laboratory of Genetic Resources and Evolution, Kunming Institute of Zoology and Kunming Primate Research Center, Chinese Academy of

Sciences, Kunming 650223, China

Full list of author information is available at the end of the article
}

differences between them, such as with regard to immune responses and disease progression. The wellknown example is that, compared with Indian rhesus macaques, simian immunodeficiency virus (SIV) pathogenesis in Chinese rhesus macaques is closer to HIV-1 infections in untreated adult humans $[9,10]$. Although previous studies have determined thousands of SNPs and hundreds of microsatellite polymorphisms [8,11-16], a genome-wide high-density genetic variation map of rhesus macaque could provide much more comprehensive information. Therefore, developing a global map of genetic variations within and between Indian- and Chinese-derived rhesus macaques has important implications for biomedical research and drug development.

Here we sequenced the genome of a male Chinese macaque and compared the data with the released reference genome of an Indian macaque (rheMac2) [2]. We identified a total of 2.94 million SNPs that are heterozygous in the Chinese macaque and 2.56 million SNPs that are different between the Chinese macaque and the reference Indian macaque genomes. We also observed

\section{Biomed Central}


123,610 deletions and other structural variations (SVs) by comparing Chinese with Indian macaques. We constructed a database, the Chinese Macaque Single Nucleotide Polymorphism (CMSNP) database, to display the SNPs and SVs using the Generic Genome Browser (GBrowse) platform. We have also integrated other valuable annotated information to enrich the CMSNP database, resulting in a comprehensive compilation of rhesus macaque genetic variations.

\section{Results and discussion Data generation}

A peripheral blood sample was collected from a healthy male Chinese rhesus macaque; this was used for DNA extraction using the standard phenol/chloroform method. We performed whole-genome sequencing of this macaque genomic DNA sample using the Illumina Genome Analyser, with span sizes of the three pairedend DNA libraries ranging from 44 to $200 \mathrm{bp}$. In total, 33 gigabases of high quality sequences with 706.5 million reads (read lengths of 44 and $49 \mathrm{bp}$ ) were generated.

Using the improved Short Oligonucleotide Alignment Program (SOAP2) [17], we mapped the reads to the reference Indian macaque genome. A summary of the resequencing data is shown in Table 1 and in Table S1 in Additional file 1. In general, $92.64 \%$ of the reads can be mapped to a unique position in the reference genome and $95.95 \%$ of the bases in the reference genome are covered, resulting in an average 11.56-fold coverage. The relatively lower genome coverage in macaque resequencing compared with the more than $99 \%$ genome coverage in human resequencing $[18,19]$ is likely due to the relatively low sequencing coverage of our study and the reference macaque genome assembly.

\section{SNP identification}

For SNP identification, we utilized a statistical model based on Bayesian algorithms that has been used in human resequencing analysis [18]. A consensus sequence (CNS) was then obtained, and a series of criteria were used to filter out the unreliable portion of the CNS for SNP detection (see Materials and methods). After filtering, a total of 5.5 million SNPs were detected (error rate $\leq 1 \%$ ), of which 2.94 million are heterozygous (two alleles are different in Chinese macaque as supported by at least four reads for each allele; Figure S1a in Additional file 1). The remaining 2.56 million SNPs are homozygous (two alleles are the same in Chinese rhesus macaque but different from Indian macaque as supported by at least four reads; Figure S1b in Additional file 1). Assuming a Poisson distribution $(\lambda=$ 11.56), the expected false discovery rate with four or more supporting reads is less than 0.001. It has been shown that the total number of SNPs would reach saturation at a sequencing depth greater than ten-fold using the paired-end reads [20]. Therefore, with 11.56fold coverage, we likely uncovered all the SNPs in the genome of the Chinese macaque individual. The observed ratio of heterozygous to homozygous SNPs is 1.18 , similar to the ratio observed for an individual human genome [21].

Compared with the previously identified 1,476 SNPs across five Encyclopedia of DNA Elements (ENCODE) regions in Chinese and Indian macaques (9 Chinese and 38 Indian rhesus macaques) [8], we completely identified 305 SNPs located in the same approximately 150-kb ENCODE regions, and $68.9 \%$ (210 of 305) of these are shared, indicating that most of the SNPs identified can be confirmed by the published dataset. Based on the shared SNPs, we conducted a hierarchical clustering analysis and the result indicates that the Chinese macaque sequenced in this study clusters with the Chinese macaques from [8] (approximately unbiased value is 94\%; Figure S2 in Additional file 1), supporting the population identity of the sequenced Chinese macaque. Additionally, our results suggest that these SNPs could efficiently distinguish Indian-derived from Chinesederived rhesus macaques [15].

The chromosomal distribution of SNPs (excluding sexual chromosomes) per $1-\mathrm{Mb}$ window is shown in Figure 1. The result indicates unbiased distribution of SNPs across the genome with a density of 2.08 SNPs per kilobase.

\section{Identification, verification and analysis of structural variation}

Paired-end sequencing is also a powerful tool for detecting genomic SV [22]. When reads are aligned onto the reference, a mated pair of reads should be in the correct orientation and the distance between them should be in an allowed range depending on the insert size of the sequenced library. If the mated pair of reads is not in a correct orientation or does not have an allowed span size, it may indicate a potential SV. We gathered abnormal mated pairs of reads for SV detection (see Materials

Table 1 Summary of the Chinese rhesus macaque re-sequencing data

\begin{tabular}{lccccccc}
\hline $\begin{array}{l}\text { Genome } \\
\text { size }\end{array}$ & $\begin{array}{c}\text { Effective } \\
\text { length }\end{array}$ & $\begin{array}{c}\text { Number of } \\
\text { reads }\end{array}$ & $\begin{array}{c}\text { Number of mapped } \\
\text { reads }\end{array}$ & $\begin{array}{c}\text { Number of } \\
\text { bases }\end{array}$ & $\begin{array}{c}\text { Number of mapped } \\
\text { bases }\end{array}$ & $\begin{array}{c}\text { Effective } \\
\text { depth }\end{array}$ & $\begin{array}{c}\text { Coverage } \\
\text { (\%) }\end{array}$ \\
\hline $2,864,106,071$ & $2,646,668,809$ & $706,459,956$ & $654,493,989$ & $33,064,980,424$ & $30,607,016,223$ & 11.56 & 95.95 \\
\hline
\end{tabular}




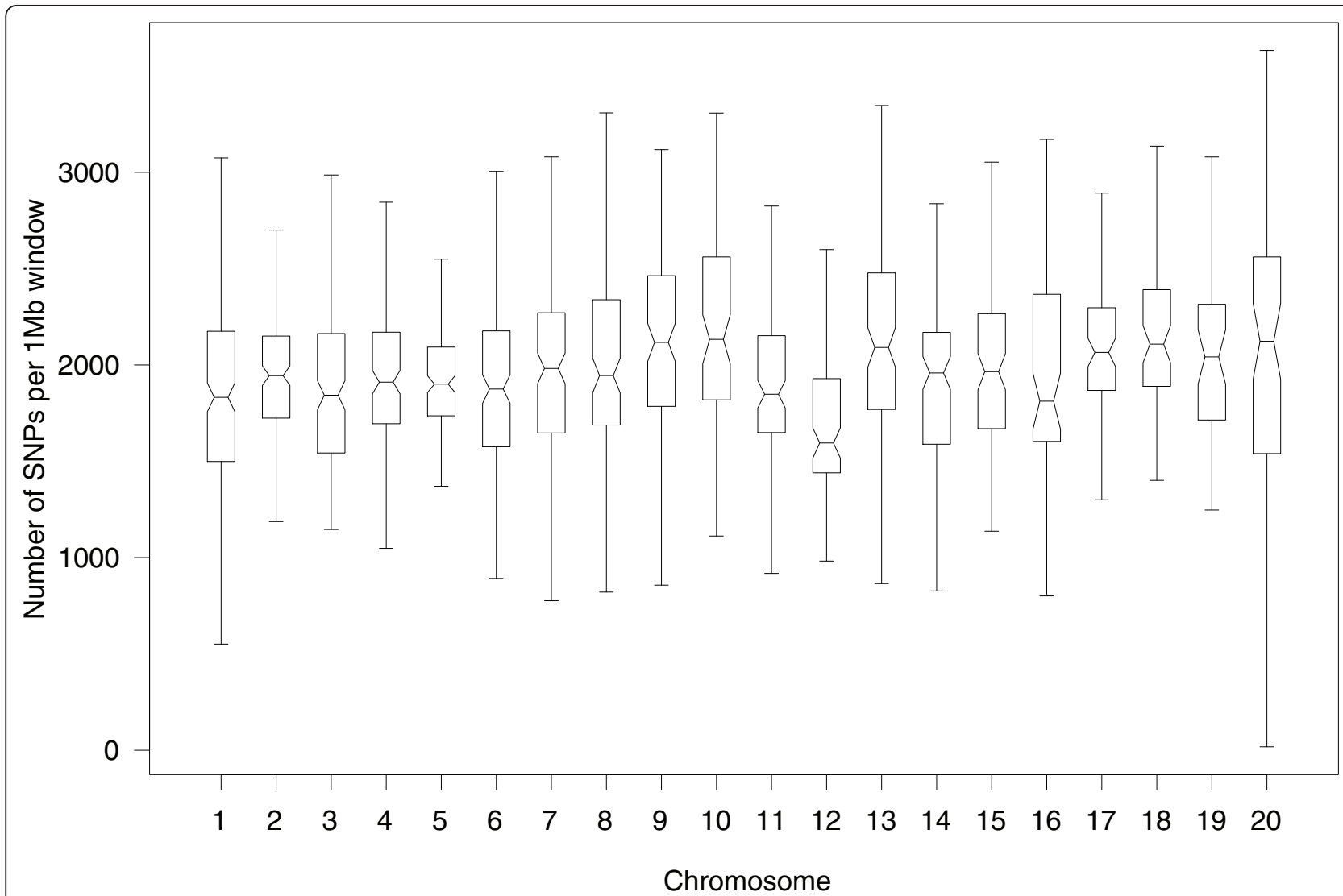

Figure 1 Chromosomal distribution of the autosomal SNPs in 1-Mb windows. Due to lower coverage (two-fold coverage), we have excluded the analysis of the sex chromosomes.

and methods). After masking unreliable SVs located in gap regions of the reference genome (the sequence alignments crossing the gap regions), a total of 125,150 SVs were identified (Figure 2a), most of which $(123,610$, $98.8 \%$ ) were deletions since deletions are easier to detect, consistent with previous reports [20,21,23]. There were 36,969 (30\%) deletions overlapping with the repeat elements in the genome, and about half of the repeat elements $(18,438)$ were Alu elements (Table S2 in Additional file 1).

To evaluate the reliability of the SVs based on our computational strategy, we focused on the deletions, which account for $98.8 \%$ of the identified SVs. We randomly selected 100 deletions (from the 123,610 candidate SVs) for PCR-based sequencing. Among them were 18 deletions located in repetitive regions in the genome that failed to be PCR amplified. The remaining 82 putative deletions were successfully amplified and sequenced; 74 of these are real deletions and the other 8 are false positive SVs (Table S3 in Additional file 2). Altogether, the deletion identification was highly accurate.

To further study the underlying mechanism of the deletions, we analyzed their length and sequence features. Based on the sequenced 74 deletions, we first compared the observed deletion lengths with the predicted pattern (Figure $2 \mathrm{~b}$ ). The length of the predicted deletion is $143 \mathrm{bp}$, on average, which is larger than that of the observed average (Table S4 in Additional file 2), likely due to the method used for identifying SVs (see Materials and methods). We further corrected the predicted deletion length and surveyed the size distribution of the deletions (Figure 2c). Genome-wide distribution of these deletions indicates that $62.8 \%$ of the deletions are located in intergenic regions and $34.5 \%$ are in intronic regions (Figure $2 \mathrm{~d}$ ). Compared with the randomly selected equivalent regions in the genome (a total of 105,000 regions with 5,000 from each of the 21 chromosomes), we observed a significant bias $(P<0.001$, Chisquared test with 1,000 replicates by Monte Carlo simulation) for intergenic regions, suggesting deletions occur more frequently in regions with low functional constraint.

We also tested whether the 74 experimentally verified deletions are polymorphic within Chinese macaque populations. We selected 20 Chinese rhesus macaques derived from four distinct geographical sites (5 from 
(a)

\begin{tabular}{ll}
\hline \multicolumn{2}{l}{ Summary of structural variations } \\
\hline Deletion & Counts \\
Insertion & 123,610 \\
Tandem duplication & 1,357 \\
Other complex & 52 \\
Total & 125,510 \\
\hline
\end{tabular}

(c)

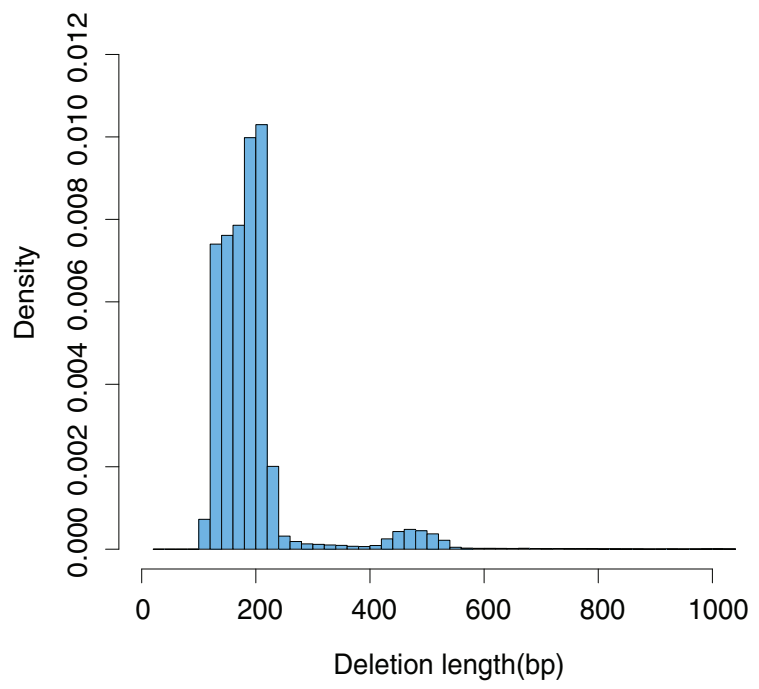

(b)

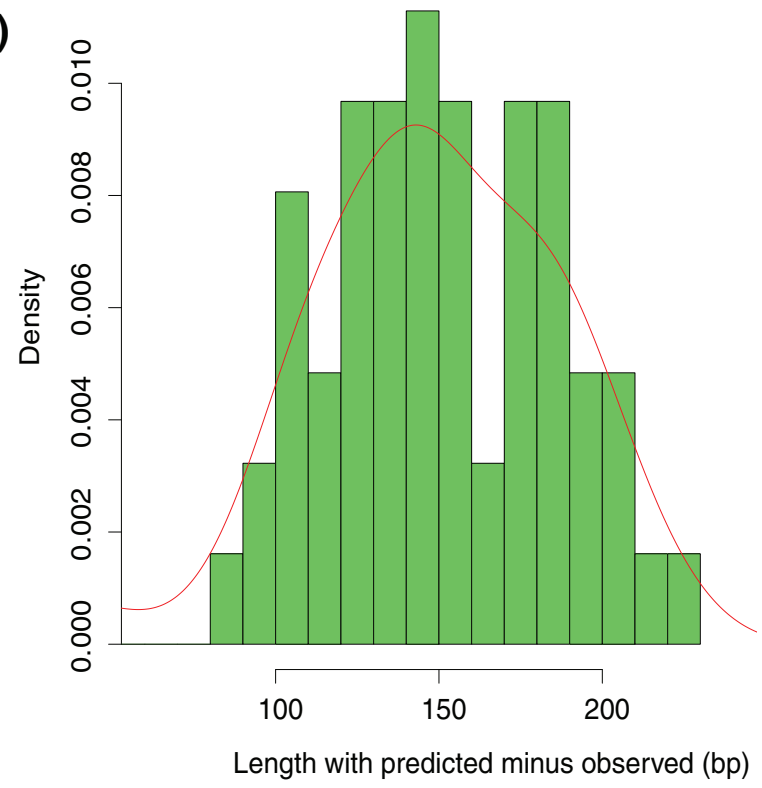

(d)

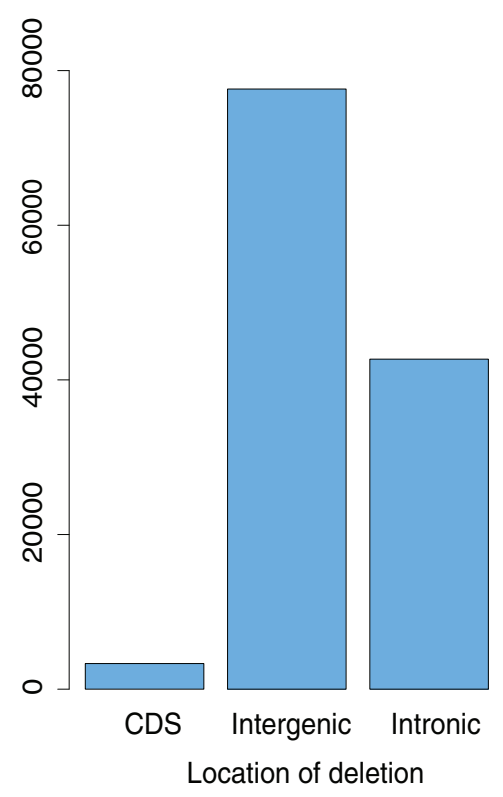

Figure 2 Summary of the identified SVs. (a) The type and number of SVs. (b) The size distribution of the predicted deletion lengths minus the observed deletion SV lengths for the experimentally tested deletion SVs. (c) The size distribution of the corrected deletion SVs. (d) The genome-wide distribution of deletions.

Sichuan Province, 5 from Yunnan Province, 5 from Guangxi Province, and 5 from Guizhou Province; Figure S3 in Additional file 1). Of the 74 deletions, 23 are fixed (homozygous), 45 are polymorphic, and the remaining 6 are uncertain (Table S5 in Additional file 2). This result suggests that a substantial portion of SVs inferred by comparing Chinese and Indian macaques are polymorphic, and those SVs fixed in Chinese macaques are particularly valuable as novel genetic markers for determining the geographic origins of macaques.

\section{Gene-based variations}

Nonsynonymous SNPs are believed to make a significant contribution to phenotypic variation within populations [24]. They are also good candidate mutations that may explain Mendelian diseases. Thus, we mapped the 5.5 
million SNPs to annotated macaque genes (Ensembl) [25] to identify the nonsynonymous SNPs in the genome; we found 43,959 SNPs in coding regions, of which 18,324 SNPs are nonsynonymous, accounting for approximately $41.7 \%$ of SNPs in the coding regions.

\section{Variations of orthologous disease and drug-target genes}

One of the primary goals of the Chinese macaque genome resequencing is to maximize the use of the rhesus macaque genome sequence in the context of biomedical research. Revealing genetic variations located in diseaserelated and drug-target genes in macaques should be helpful to this purpose.

Our preliminary analysis identified a total of 6,823 macaque orthologs of human disease genes, of which 4,558 orthologs have at least one SNP in the coding regions, and 2,462 orthologs have at least one nonsynonymous SNP (Figure 3a). Overall, we observed 15,005 SNPs within the coding regions of these genes, of which 9,818 are synonymous and 5,187 are nonsynonymous. Additionally, we analyzed SV distribution in the 6,823 macaque disease orthologs. A total of 4,508 orthologous macaque disease genes bear at least one SV and there are 20,775 SVs within these genes (approximately $88.9 \%$ of SVs are located in introns).

We also performed a similar analysis on the drug-target genes. Genetic variants within the drug-target genes would have the potential to influence drug effects and could be a valuable resource for pharmacogenomic study. We mapped the variants to the macaque orthologs of human drug-target genes downloaded from DrugBank [26]. A total of 954 orthologous macaque drug-target genes have at least one SNP in the coding regions, and 483 of them bear at least one nonsynonymous SNPs (Figure 3B). Overall, we observed 2,980 SNPs within the coding regions of these genes, and 962 of them are nonsynonymous and 2,018 are synonymous. In addition, the 949 orthologous macaque drug-target genes have at least one SV in the genomic regions and there are 4,091 SVs within these genes.

Protein domains form functional units that are often the targets of drugs; these are called 'druggable domains' [27]. Thus, nonsynonymous SNPs within druggable domains are more likely correlated with clinical variations during drug treatment. To study this, we used 962 identified nonsynonymous SNPs within the coding regions of 483 macaque drug-target orthologs to identify SNPs within the druggable domains (see Materials and methods). A total of 478 nonsynonymous SNPs located in 273 unique genes were identified in the druggable domains (Table S6 in Additional file 3). Meanwhile, to detect whether these SNP-containing druggable domains in the macaque drug-target orthologs also have SNPs in their human counterparts, PolyDoms, a previously developed database that maps all coding SNPs in protein domains [28], was used to search for SNPs located in the same domains using macaque orthologs as the query. In total, 671 unique nonsynonymous SNPs were discovered in the same druggable domains (Table S6 in Additional file 3). These shared druggable domain SNPs between Chinese macaque and human provide a highly useful tool to access between-individual drug treatment variations in preclinical trials using macaques.

\section{The Chinese macaque genetic variation database}

We have established the Chinese Macaque Single Nucleotide Polymorphism (CMSNP) database [29] for data visualization. We integrated our variation and other associated data into Gbrowse, a popular genome browser used in the GMOD project [30] (Table 2). We have also integrated annotated macaque genes [25] and microRNAs [31] into the CMSNP database for the purpose of understanding genetic variations at the gene level, as well as orthologous macaque disease and drugtarget genes, which is helpful to further biomedical research. Finally, the evolutionarily conserved regions between human and macaque were added in Gbrowse [32], which can be used to understand the genetic variations within these conserved regions. All data have been organized into a MySQL relational database, which is efficient in retrieving data from indexed files.

The CMSNP database is loaded in large batches and used primarily in read-only mode. An overview of the browser window is shown in Figure 4. The query forms supported in the CMSNP database include gene nomenclature, sequence coordinates, and CMSNP IDs, which are recorded by appending seven numbers (for example, CMSNP0000001). Individual entries within a track have either associated internal pages that provide information about the annotation or related links to external sites and databases.

\section{Conclusions}

The variation map of rhesus macaque provides a useful framework for further genome-wide association studies and also has important applications to evolutionary and functional studies.

\section{Materials and methods DNA sequencing}

Genomic DNA was purified from a 4-year-old male Chinese rhesus macaque from Sichuan Province of China. The standard phenol-chloroform method was used for DNA extraction.

The genomic DNA was fragmented by nebulization with compressed nitrogen gas. The overhangs of the fragments were converted to blunt ends using T4 DNA polymerase and Klenow polymerase. After adding an ' $\mathrm{A}$ ' 
(a)

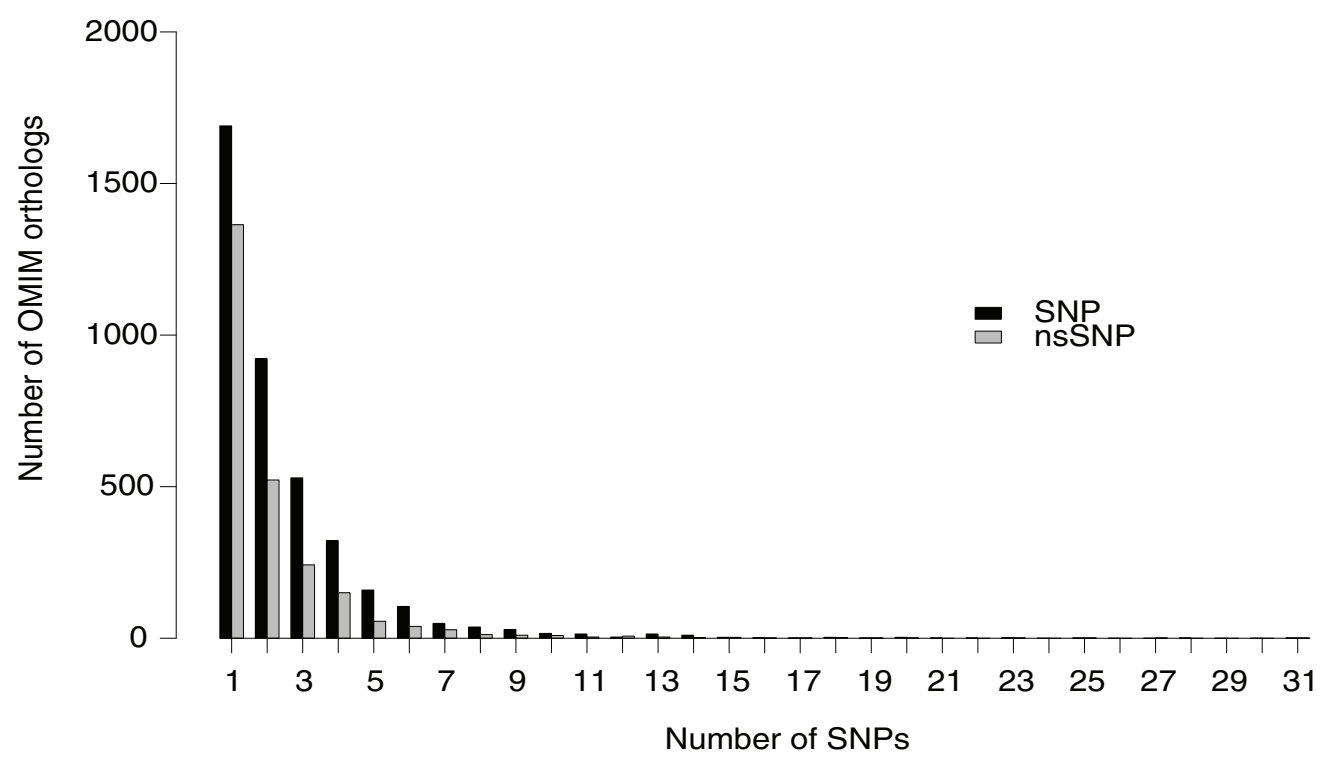

(b)

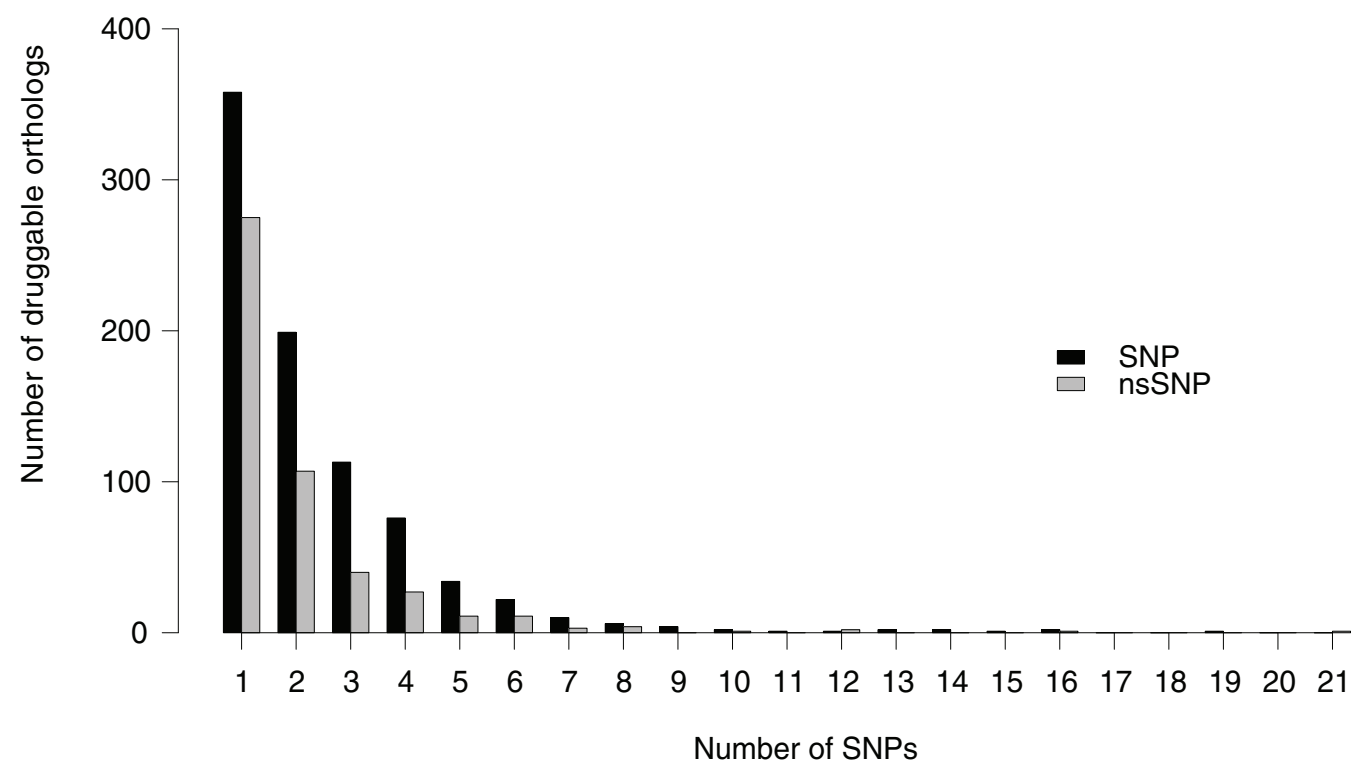

Figure 3 Distribution of variants in the orthologous macaque disease and drug-target genes. (a) The distribution of orthologous disease genes that contain one or more SNPs in their coding regions. (b) The distribution of the orthologous drug-target genes that contain one or more SNPs in their coding regions. SNP and nsSNP denote synonymous and non-synonymous SNPS, respectively. OMIM, Online Mendelian Inheritance in Man. 
Table 2 Datasets integrated into the CMSNP database

\begin{tabular}{ll}
\hline Datasets & Source \\
\hline SNPs & Sequencing \\
SVs & Sequencing \\
Macaque genome sequence & NCBI database \\
OMIM orthologs & OMIM database [33] \\
Drug-target orthologs & DrugBank database [26] \\
Macaque annotated genes & Ensembl database (release 51) [25] \\
Macaque microRNAs & Sanger mirBase (release 12.0) [31] \\
Evolutionarily conserved elements & ECRBase [32] \\
Promoter dataset & ECRBase \\
Synteny dataset & ECRBase \\
\hline
\end{tabular}

OMIM, Online Mendelian Inheritance in Man.

base to the blunt ends of the double-stranded DNA fragments, adaptors with ' $\mathrm{T}$ ' base overhangs were ligated to the genomic DNA fragments. These fragments were separated on an agarose gel and excised from the gel at the DNA band around $200 \mathrm{bp}$. Finally, the DNA fragments were enriched by a ten-cycle PCR process.

DNA sequencing was performed using an Illumina Genome Analyser (Solexa, San Diego, California USA) according to the manufacturer's instructions. Fluorescent image deconvolution, quality value calculation and sequence conversion were carried out using the Illumina base-calling pipeline.

\section{SNP and structural variation identification}

All sequenced reads were aligned to the reference rhesus macaque genome (rheMac2) using SOAP2 [17], with two mismatches allowed. After alignment, we used a statistical model based on Bayesian theory and the Illumina quality system to calculate the probability of each possible genotype of each position on the reference genome. At each position, the genotype was called by the highest probability, and a CNS was then obtained. The final CNS probabilities were transformed to quality scores in the Phred scale.

SNPs were obtained by a combination of parameters set to filter the CNS. The candidate SNPs were extracted from the CNS and then filtered using defined criteria to obtain the final SNP set. The filter criteria used included a Q20 quality cutoff (quality score $\geq 20$ or error rate $<1 \%$ ), estimated copy number of flanking sequences $(<2)$, minimum distance of two given SNPs $(\geq 5 \mathrm{bp})$, and overall depth $(\leq 100)$ in a given position in the reference. For both homozygous and heterozygous SNPs we required the support of at least four reads for each allele. Using cumulative Poisson statistics $(\lambda=11.56)$, the expected false discovery rate with four or more supported reads is less than 0.001. We compared the called SNPs in this study with previously identified SNPs across five ENCODE regions for data evaluation. Hierarchical clustering analysis with 1,000 bootstraps based on the locally shared SNPs was conducted to determine the population identity of the sequenced Chinese macaque. We also determined the chromosomal distribution of SNPs (excluding sexual chromosomes due to half coverage compared with autosomes) using 1-Mb windows.

According to the span size between the mapped paired-end reads and their orientations, alignments are divided into two types. The first type is the normal mated pair, which has the correct orientation and an allowed span size, and the other type is defined as an abnormal mated pair, which can be used for SV detection. SVs were called if the lengths were more than three times the standard deviation of the insert size of the DNA library. The insert sizes of all libraries constructed were $200 \mathrm{bp}$. For SV identification, we grouped the abnormal read pairs into diagnostic paired-end clusters. In order to avoid misalignment, each detected SV should be supported by at least four reads. We then examined and organized the SVs into alignment models, including deletion, insertion, inversion, translocation, duplication, and so on. Different types of SVs have a predefined mated pair alignment pattern that is inferred from the Solexa sequencing technology. For example, if there is a deletion in the sequenced individual, the mated pair of reads across the break point may have an abnormal span size but the correct orientation when aligned to the reference. SVs that overlap another SV in a spanned region were defined as complex SVs.

\section{Verification of structural variation}

To verify SV, we randomly chose 100 deletions. Primers were designed by using the deletion region and the flanking 150-bp sequences (primers are listed Table S3 in Additional file 2). In addition, we also tested whether these deletions are polymorphic by screening 20 Chinese rhesus macaques from different geographic origins. All the 20 Chinese rhesus macaques were males and the blood samples were obtained from Kunming Primate Research Center, Chinese Academy of Sciences. DNA was isolated by the standard phenol-chloroform method.

\section{SNPs in disease genes}

The identification of disease gene orthologs in the macaque genome was conducted through canonically reciprocal best-to-best hits implemented in the BLASTP program with default parameters between human proteins encoded by disease genes compiled from the Online Mendelian Inheritance in Man database [33] and macaque annotated proteins (for each gene, the longest transcript was selected); all synonymous and nonsynonymous SNPs were then annotated and assigned to macaque orthologs of human disease genes. 


\section{(a)}

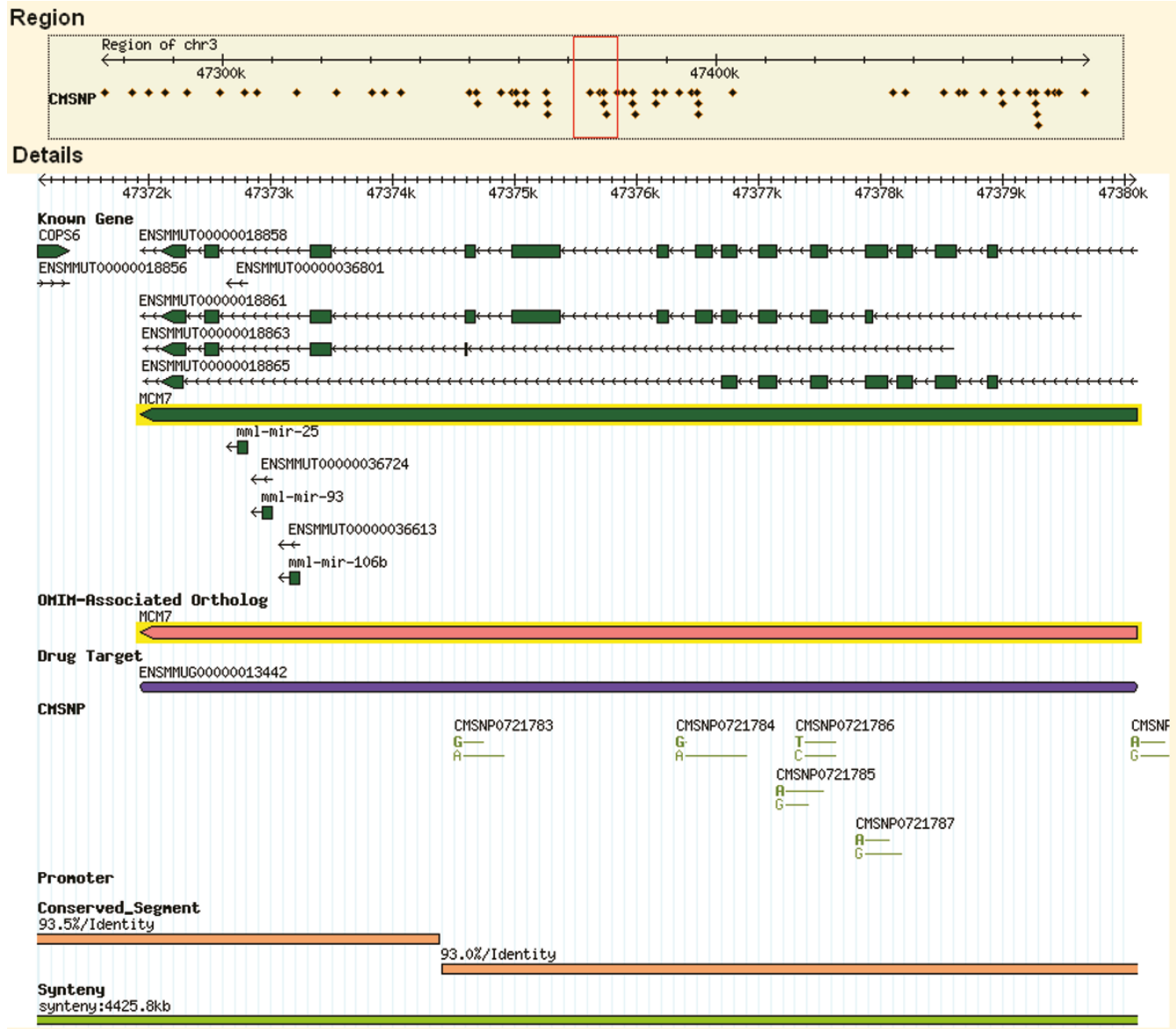

(b)

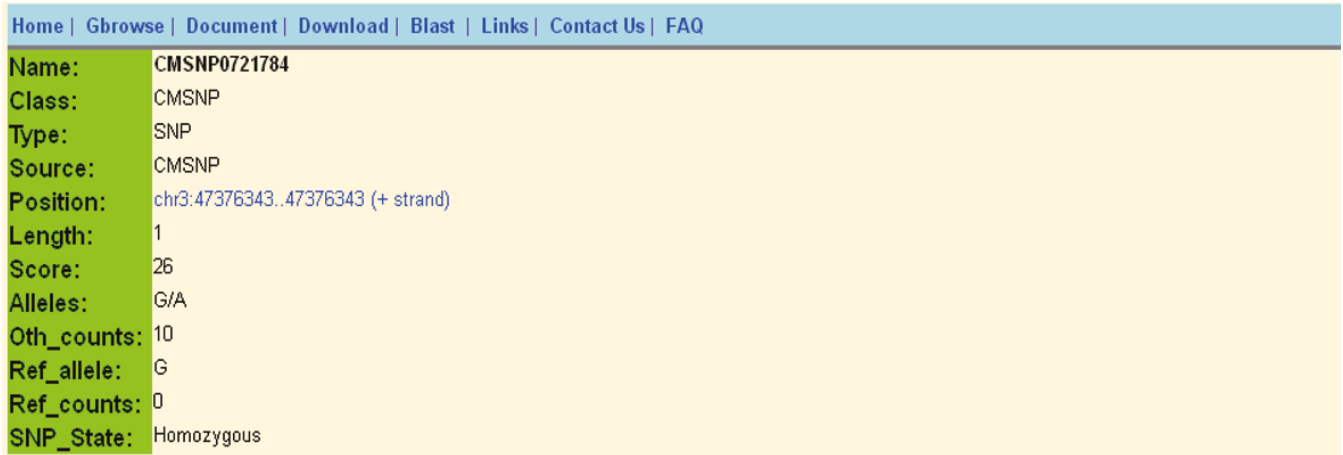

Figure 4 Screenshots of the browser window of a specific region. (a) The Region panel is an overview of SNP distribution in a specific region and SNPs are displayed as a glyph of triangles. The detailed panel shows the types and counts of SNPs, and the bold green-colored type is the reference allele. Other tracks, such as biomedical associations and conservation, are also displayed with glyphs and colors. (b) More detailed descriptions of each SNP are linked for viewing. 
SNPs in druggable protein domains

For identification of nonsynonymous SNPs within druggable protein domains, first all orthologous macaque druggable protein targets were identified through canonically reciprocal best-to-best hits implemented in the BLASTP program with default parameters between human druggable protein targets downloaded from the DrugBank database [26] and macaque annotated proteins (for each gene, the longest length of CDS was selected). A series of Perl scripts were then parsed to identify 483 nonsynonymous SNP-containing druggable orthologs. Finally, based on druggable human protein domains documented in the DrugBank database, human druggable protein targets were blatted against protein domain data downloaded from the Pfam (23.0) database [34] to identify the location of druggable domains; MUSCLE [35] alignment followed by Perl scripts were used to extract the corresponding nonsynonymous-SNPcontaining druggable domains.

To identify the human SNPs within the domains detected in macaque, we used 273 gene symbols as queries to search in the PolyDoms database, which integrates all coding SNPs in human protein domains.

\section{Database construction}

The CMSNP database contains two main datasets, an SNP dataset and an SV dataset, which are generated from our resequencing data. The annotated macaque gene dataset and the known macaque microRNA dataset were obtained from the Ensembl Biomart (release 51) [25] and the Sanger miRBase (release 12) [31], respectively. We also downloaded three additional datasets from the evolutionarily conserved regions database (ECRBase) [32], including the promoter dataset, the synteny dataset (between human and macaque), and the evolutionarily conserved region dataset.

A series of Perl scripts were used to convert these datasets into the GFF (General Feature Format) file format. Then, as the Gbrowse tutorial [36] recommends, we used bp_load_gff.pl to import all GFF-formatted files into the MySQL relational database.

\section{Data accessibility}

The sequence data have been deposited in the NCBI Short Read Archive [37] under accession number [SRA037810].

\section{Additional material}

Additional file 1: Tables S1 and S2 and Figures S1 to S3. Table S1: detailed summary of Chinese rhesus macaque resequencing data. Table S2: summary of the overlapping deletions with repeat elements. Figure S1: cumulative density of read counts for homozygous and heterozygous SNPs. Figure S2: hierarchical clustering of rhesus macaques. Figure S3: distribution of the 20 Chinese rhesus macaques used for SV polymorphism testing.

Additional file 2: Supplementary tables. Table S3: information for selected SVs and primers used for PCR and sequencing. Table S4: deletion length information. Table S5: SV status in Chinese macaque population.

Additional file 3: Table S6. SNPs in drug-target protein domains

\section{Abbreviations}

bp: base pair; CMSNP: Chinese Macaque Single Nucleotide Polymorphism; CNS: consensus sequence; ENCODE: Encyclopedia of DNA Elements; PCR: polymerase chain reaction; SNP: single nucleotide polymorphism; SV: structural variation.

\section{Acknowledgements}

We thank Yanjiao Li for her help with DNA isolation and Chao Song for his assistance in database construction. We also thank Drs Ryan D Hernandez and Carlos D Bustamante for kindly providing the SNP data of the five ENCODE regions. This study was supported by the National 973 project of China (2011CBA01101) and the National Natural Science Foundation of China (30871343).

\section{Author details}

${ }^{1}$ Beijing Genomics Institute-Shenzhen, Chinese Academy of Sciences, Shenzhen 518083, China. ${ }^{2}$ State Key Laboratory of Genetic Resources and Evolution, Kunming Institute of Zoology and Kunming Primate Research Center, Chinese Academy of Sciences, Kunming 650223, China. ${ }^{3}$ Graduate School of Chinese Academy of Sciences, Beijing 100039, China. ${ }^{4}$ Current address: Beijing Institute of Genomics, Chinese Academy of Sciences, Beijing 100029, China.

\section{Authors' contributions}

BS and JW designed the study; XF, YZ, RZ, XG LY, ML and KY carried out sequencing and data analysis; $X F, Y Z, R Z$, JW and BS wrote the manuscript. All authors read and approved the final manuscript.

Received: 14 December 2010 Revised: 1 May 2011

Accepted: 6 July 2011 Published: 6 July 2011

\section{References}

1. Goodman M, Porter CA, Czelusniak J, Page SL, Schneider H, Shoshani J, Gunnell G, Groves CP: Toward a phylogenetic classification of primates based on DNA evidence complemented by fossil evidence. $\mathrm{Mol}$ Phylogenet Evol 1998, 9:585-598.

2. Gibbs RA, Rogers J, Katze MG, Bumgarner R, Weinstock GM, Mardis ER, Remington KA, Strausberg RL, Venter JC, Wilson RK, Batzer MA, Bustamante CD, Eichler EE, Hahn MW, Hardison RC, Makova KD, Miller W, Milosavljevic A, Palermo RE, Siepel A, Sikela JM, Attaway T, Bell S, Bernard KE, Buhay CJ, Chandrabose MN, Dao M, Davis C, Delehaunty KD, Ding $Y$, et al: Evolutionary and biomedical insights from the rhesus macaque genome. Science 2007, 316:222-234.

3. Colman RJ, Anderson RM, Johnson SC, Kastman EK, Kosmatka KJ, Beasley TM, Allison DB, Cruzen C, Simmons HA, Kemnitz JW, Weindruch R: Caloric restriction delays disease onset and mortality in rhesus monkeys. Science 2009, 325:201-204.

4. Weiss RA: Polio vaccines exonerated. Nature 2001, 410:1035-1036.

5. Yang SH, Cheng PH, Banta H, Piotrowska-Nitsche K, Yang JJ, Cheng EC, Snyder B, Larkin K, Liu J, Orkin J, Fang ZH, Smith Y, Bachevalier J, Zola SM, Li SH, Li XJ, Chan AW: Towards a transgenic model of Huntington's disease in a non-human primate. Nature 2008, 453:921-924.

6. Chan AW, Yang SH: Generation of transgenic monkeys with human inherited genetic disease. Methods 2009, 49:78-84.

7. Disotell TR, Tosi AJ: The monkey's perspective. Genome Biol 2007, 8:226.

8. Hernandez RD, Hubisz MJ, Wheeler DA, Smith DG, Ferguson B, Rogers J, Nazareth L, Indap A, Bourquin T, McPherson J, Muzny D, Gibbs R, Nielsen R, Bustamante CD: Demographic histories and patterns of linkage 
disequilibrium in Chinese and Indian rhesus macaques. Science 2007, 316:240-243.

9. Trichel AM, Rajakumar PA, Murphey-Corb M: Species-specific variation in SIV disease progression between Chinese and Indian subspecies of rhesus macaque. J Med Primatol 2002, 31:171-178.

10. Ling B, Veazey RS, Luckay A, Penedo C, Xu K, Lifson JD, Marx PA: SIV(mac) pathogenesis in rhesus macaques of Chinese and Indian origin compared with primary HIV infections in humans. Aids 2002, 16:1489-1496.

11. Satkoski J, Malhi RS, Kanthaswamy S, Tito RY, Malladi VS, Smith DG: Pyrosequencing as a method for SNP identification in the rhesus macaque (Macaca mulatta). BMC Genomics 2008, 9:256.

12. Rogers J, Garcia R, Shelledy W, Kaplan J, Arya A, Johnson Z, Bergstrom M, Novakowski L, Nair P, Vinson A, Newman D, Heckman G, Cameron J: An initial genetic linkage map of the rhesus macaque (Macaca mulatta) genome using human microsatellite loci. Genomics 2006, 87:30-38.

13. Malhi RS, Sickler B, Lin D, Satkoski J, Tito RY, George D, Kanthaswamy S, Smith DG: MamuSNP: a resource for rhesus macaque (Macaca mulatta) genomics. PLOS ONE 2007, 2:e438.

14. Raveendran M, Harris RA, Milosavljevic A, Johnson Z, Shelledy W, Cameron J, Rogers J: Designing new microsatellite markers for linkage and population genetic analyses in rhesus macaques and other nonhuman primates. Genomics 2006, 88:706-710.

15. Ferguson B, Street SI, Wright $H$, Pearson C, Jia Y, Thompson SL, Allibone P, Dubay CJ, Spindel E, Norgren RB: Single nucleotide polymorphism (SNPs) distinguish Indian-origin and Chinese-origin rhesus macaques (Macaca mulatta). BMC Genomics 2007, 8:43.

16. Khouangsathiene S, Pearson C, Street S, Ferguson B, Dubay C: MonkeySNP: a web portal for non-human primate single nucleotide polymorphisms. Bioinformatics 2008, 24:2645-2646.

17. Li R, Li Y, Kristiansen K, Wang J: SOAP: short oligonucleotide alignment program. Bioinformatics 2008, 24:713-714.

18. Wang J, Wang W, Li R, Li Y, Tian G, Goodman L, Fan W, Zhang J, Li J, Zhang J, Guo Y, Feng B, Li H, Lu Y, Fang X, Liang H, Du Z, Li D, Zhao Y, Hu Y, Yang Z, Zheng H, Hellmann I, Inouye M, Pool J, Yi X, Zhao J, Duan J, Zhou Y, Qin J, et al: The diploid genome sequence of an Asian individual. Nature 2008, 456:60-65

19. Tong P, Prendergast JG, Lohan AJ, Farrington SM, Cronin S, Friel N, Bradley DG, Hardiman O, Evans A, Wilson JF, Loftus B: Sequencing and analysis of an Irish human genome. Genome Biol 2010, 11:R91.

20. Wang J, Wang W, Li R, Li Y, Tian G, Goodman L, Fan W, Zhang J, Li J, Guo Y, Feng B, Li H, Lu Y, Fang X, Liang H, Du Z, Li D, Zhao Y, Hu Y, Yang Z, Zheng H, Hellmann I, Inouye M, Pool J, Yi X, Zhao J, Duan J, Zhou Y, Qin J, Ma L, et al: The diploid genome sequence of an Asian individual. Nature 2008, 456:60-65.

21. Levy S, Sutton G, Ng PC, Feuk L, Halpern AL, Walenz BP, Axelrod N, Huang J, Kirkness EF, Denisov G, Lin Y, MacDonald JR, Pang AW, Shago M, Stockwell TB, Tsiamouri A, Bafna V, Bansal V, Kravitz SA, Busam DA, Beeson KY, Mclntosh TC, Remington KA, Abril JF, Gill J, Borman J, Rogers YH, Frazier ME, Scherer SW, Strausberg RL, et al: The diploid genome sequence of an individual human. PLOS Biol 2007, 5:e254

22. Metzker ML: Sequencing technologies - the next generation. Nat Rev Genet 2010, 11:31-46.

23. McKernan K, Peckham HE, Costa GL, McLaughlin SF, Fu Y, Tsung EF, Clouser CR, Duncan C, Ichikawa JK, Lee CC, Zhang Z, Ranade SS, Dimalanta ET, Hyland FC, Sokolsky TD, Zhang L, Sheridan A, Fu H, Hendrickson CL, Li B, Kotler L, Stuart JR, Malek JA, Manning JM, Antipova AA, Perez DS, Moore MP, Hayashibara KC, Lyons MR, Beaudoin RE, et al: Sequence and structural variation in a human genome uncovered by short-read, massively parallel ligation sequencing using two-base encoding. Genome Res 2009, 19:1527-1541.

24. Chun S, Fay JC: Identification of deleterious mutations within three human genomes. Genome Res 2009, 19:1553-1561.

25. Hubbard TJ, Aken BL, Ayling S, Ballester B, Beal K, Bragin E, Brent S, Chen Y, Clapham P, Clarke L, Coates G, Fairley S, Fitzgerald S, Fernandez-Banet J, Gordon L, Graf S, Haider S, Hammond M, Holland R, Howe K, Jenkinson A, Johnson N, Kahari A, Keefe D, Keenan S, Kinsella R, Kokocinski F, Kulesha E, Lawson D, Longden I, et al: Ensembl 2009. Nucleic Acids Res 2009, 37 D690-697.
26. Wishart DS, Knox C, Guo AC, Cheng D, Shrivastava S, Tzur D, Gautam B, Hassanali M: DrugBank: a knowledgebase for drugs, drug actions and drug targets. Nucleic Acids Res 2008, 36:D901-906.

27. Overington JP, Al-Lazikani B, Hopkins AL: How many drug targets are there?. Nat Rev Drug Discov 2006, 5:993-996.

28. Jegga AG, Gowrisankar S, Chen J, Aronow BJ: PolyDoms: a whole genome database for the identification of non-synonymous coding SNPs with the potential to impact disease. Nucleic Acids Res 2007, 35:D700-706.

29. CMSNP.. [http://monkey.genomics.org.cn/].

30. Stein LD, Mungall C, Shu S, Caudy M, Mangone M, Day A, Nickerson E, Stajich JE, Harris TW, Arva A, Lewis S: The generic genome browser: a building block for a model organism system database. Genome Res 2002, 12:1599-1610.

31. Griffiths-Jones $S$, Saini HK, van Dongen S, Enright AJ: miRBase: tools for microRNA genomics. Nucleic Acids Res 2008, 36:D154-158.

32. Loots G, Ovcharenko I: ECRbase: database of evolutionary conserved regions, promoters, and transcription factor binding sites in vertebrate genomes. Bioinformatics 2007, 23:122-124.

33. Hamosh A, Scott AF, Amberger JS, Bocchini CA, McKusick VA: Online Mendelian Inheritance in Man (OMIM), a knowledgebase of human genes and genetic disorders. Nucleic Acids Res 2005, 33:D514-517.

34. Finn RD, Tate J, Mistry J, Coggill PC, Sammut SJ, Hotz HR, Ceric G, Forslund K, Eddy SR, Sonnhammer EL, Bateman A: The Pfam protein families database. Nucleic Acids Res 2008, 36:D281-288.

35. Edgar RC: MUSCLE: multiple sequence alignment with high accuracy and high throughput. Nucleic Acids Res 2004, 32:1792-1797.

36. Gbrowse.. [http://gmod.org/wiki/Gbrowse].

37. Sequence Read Archive.. [http://trace.ncbi.nlm.nih.gov/Traces/sra/sra.cgi].

doi:10.1186/gb-2011-12-7-r63

Cite this article as: Fang et al:: Genome sequence and global sequence variation map with 5.5 million SNPs in Chinese rhesus macaque. Genome Biology 2011 12:R63.

\section{Submit your next manuscript to BioMed Central and take full advantage of:}

- Convenient online submission

- Thorough peer review

- No space constraints or color figure charges

- Immediate publication on acceptance

- Inclusion in PubMed, CAS, Scopus and Google Scholar

- Research which is freely available for redistribution

Submit your manuscript at www.biomedcentral.com/submit
Biomed Central 